\title{
A atuação do farmacêutico clínico como interventor na identificação de problemas relacionados a medicamentos em hospitais: uma revisão sistemática
}

\author{
The role of the clinical pharmacist as an intervener in the identification \\ of drug-related problems in hospitals: a systematic review
}

Recebido em: 23/11/2020 Aceito em: $\quad 02 / 02 / 2021$
Hugo dos Santos SOUZA ${ }^{1}$; Tainá Sousa GONZAGA ${ }^{1}$; Vanessa Kele Pureza BARROS ${ }^{1}$; Carla de Castro SANT'ANNA ${ }^{1}$; Marcella Kelly Costa de ALMEIDA ${ }^{2}$ ${ }^{1}$ Universidade da Amazônia - UNAMA. Av. Alcindo Cacela, 287, CEP 66060-000. Umarizal, Belém, PA, Brasil. ${ }^{2}$ Núcleo de Pesquisa em Oncologia, Universidade Federal do Pará - UFPA. R. Augusto Corrêa, 01, Guamá, CEP 66075-110. Belém, PA, Brasil. E-mail:hugo.santos23@outlook.com

\section{ABSTRACT}

Drug-related problems (PRM) are a serious problem that interferes with the efficiency of therapeutic results, with, in most cases, unwanted effects. They are responsible for increased length of stay, morbidity, mortality, and increased costs in hospitals. Thus, this research investigates the clinical pharmacist's role in identifying MRPs in hospitals, identifying the most common problems, and verifying which pharmaceutical interventions are most performed about these. A systematic review was carried out, using articles from national and international literature. The articles used in this work were published from 2015 to 2020, indexed in the MEDLINE, SciELO, LILACS, and PUBMED databases. Such a review was carried out following the guidelines of PRISMA. The search strategy identified 234 articles, leaving 15 studies for reading in full text and included in this review. It was concluded that the pharmacist works in partnership with the multi-professional team present in the hospital environment, mostly because he is the most suitable professional to solve problems related to drugs through the verification of drug treatment, evaluation of prescriptions, and pharmacotherapeutic guidance.

Keywords: Pharmaceutical services; Medication errors; Inappropriate prescribing.

\section{RESUMO}

Os problemas relacionados aos medicamentos (PRM) constituem um sério problema que interfere na eficiência dos resultados terapêuticos, apresentando, na maioria dos casos, efeitos indesejados. Eles são responsáveis por aumento no tempo de internação, morbidade, mortalidade e aumento de custos em hospitais. Desse modo, esta pesquisa tem como objetivo investigar a atuação do Farmacêutico Clínico na identificação de PRM em hospitais, identificando os problemas que são mais comuns e verificando quais as intervenções farmacêuticas mais realizadas em relação a estes. Foi realizada uma revisão sistemática, 
utilizando artigos da literatura nacional e internacional. Os artigos usados neste trabalho foram publicados no período de 2015 a 2020, indexados nos bancos de dados MEDLINE, SciELO, LILACS e PUBMED. Tal revisão foi realizada seguindo as diretrizes do PRISMA. A estratégia de busca identificou 234 artigos, deixando 15 estudos para leitura em texto completo e incluídos nesta revisão. O farmacêutico atua em parceria com a equipe multiprofissional presente no ambiente hospitalar, especialmente, por ser o profissional mais indicado para solucionar problemas relacionados a medicamentos, por meio da verificação do tratamento medicamentoso, avaliação das prescrições e orientação farmacoterapêutica.

Palavras-chave: assistência farmacêutica; erros de medicação; prescrição inadequada.

\section{INTRODUÇÃO}

Pacientes em condições críticas de saúde estão sujeitos a qualquer tipo de problema relacionado a medicamentos (PRM), principalmente, em virtude da complexidade do regime terapêutico ao qual se submetem. Segundo o Pharmaceutical Care Network Europe (PCNE), PRM é considerado qualquer evento que possa interferir na farmacoterapia do paciente, acarretando desfechos clínicos indesejáveis; tal definição engloba tanto problemas relacionados aos erros nos processos terapêuticos quanto o efeito nocivo e inesperado do medicamento $(1,2)$.

Alguns PRM referentes, por exemplo, à indicação, efetividade, segurança e adesão, podem resultar em elevado impacto social, no âmbito clínico, humanístico e econômico (3). Além disso, tais problemas estão associados a uma das principais causas de eventos adversos, podendo ser apontados como agentes causadores do aumento no tempo de internação, aumento de morbidade e/ou mortalidade e aumento de custos hospitalares (4).

Isso se dá devido à configuração do processo que envolve o uso de medicamentos, considerado de alta complexidade e que ocorre em três etapas: a prescrição, a dispensação e a administração (5). Nesse contexto, o Farmacêutico Clínico (FC) pode desenvolver um trabalho importante, que é de atuar na prevenção e redução de danos ao paciente, relacionados ao uso de medicamentos, especialmente aqueles que envolvem os erros de prescrição, ilegibilidade, nome incorreto, dosagem inadequada ou ausência dela, e erros na forma farmacêutica; estes são fatores prevalentes, que costumam ocorrer no ambiente hospitalar $(6,7)$.
No âmbito hospitalar, o farmacêutico pode favorecer a prática do uso racional de medicamentos, contribuindo para a diminuição do tempo de internação, para uma melhor qualidade de vida, para a redução do índice de mortalidade e, consequentemente, impactando de forma positiva no custo gerado (farmaeconômico) durante o período de internação (8).

Diante desse cenário, o presente estudo teve como objetivo analisar a atuação do Farmacêutico Clínico na identificação de PRM em hospitais, identificar os PRM mais citados na literatura científica e verificar as intervenções farmacêuticas mais frequentemente realizadas em relação a estes.

\section{MÉTODO}

Foi realizada uma revisão sistemática acerca da atuação do Farmacêutico Clínico como interventor na identificação de problemas relacionados a medicamentos em hospitais, utilizando artigos da literatura nacional e internacional. Tal revisão foi realizada seguindo as diretrizes do PRISMA (9).

Os artigos discutidos nesse trabalho estão indexados nas bases de dados da Medical Literature Analysis and Retrieval System Online (MEDLINE), Scientific Electronic Library Online (SciELO), Literatura Latino-Americana e do Caribe em Ciências da Saúde (LILACS), US National Library of Medicine National Institutes of Health (PUBMED), a partir dos descritores específicos (em português e inglês): assistência farmacêutica (pharmaceutical services), erros de medicação (medication errors), prescrição inadequada (inappropriate prescribing). 
Foram incluídos estudos retrospectivos e prospectivos que abordassem a atuação do Farmacêutico Clínico na identificação de problemas relacionados a medicamentos em hospitais e intervenções farmacêuticas, publicados em inglês e português, com texto completo, com publicação entre 2015 e 2020. Foram excluídos da pesquisa trabalhos publicados em anais de congresso, artigos publicados em revistas não indexadas em banco de dados selecionados, fora do período de seleção e em duplicata.

A estratégia de busca identificou 234 artigos, desse total foram excluídos 167 por não se enquadrarem no período de publicação estipulado, 3 excluídos pelo idioma espanhol e 5 duplicados, resultando em 59 para avaliação do título e resumo. Nesta fase, foram excluídos 44 artigos que não se adequaram aos objetivos desta pesquisa, deixando 15 estudos para leitura em texto completo e incluídos nesta revisão (Figura 1).

\section{RESULTADOS E DISCUSSÃO}

A integração do FC na equipe multiprofissional de assistência à saúde tem se tornado uma vertente cada vez mais presente nos sistemas de saúde, principalmente, pelos resultados positivos proporcionados pelas intervenções farmacêuticas, seja no âmbito farmacoterapêutico ou farmacoeconômico (3).

Partindo disso, os PRMs foram definidos pelo II Consenso de Granada como aqueles que geram resultados clínicos negativos, derivados da farmacoterapia que, produzidos por diversas causas, interferem no resultado terapêutico ou levam a efeitos indesejados. Tais problemas podem ser caracterizados como Erros de Medicação (EM) e como Reações Adversas a Medicamentos (RAMs); no primeiro item, são relacionados a erros que podem ocorrer durante o processo de prescrição e

Figura 1. Fluxograma dos critérios aplicados na seleção dos artigos

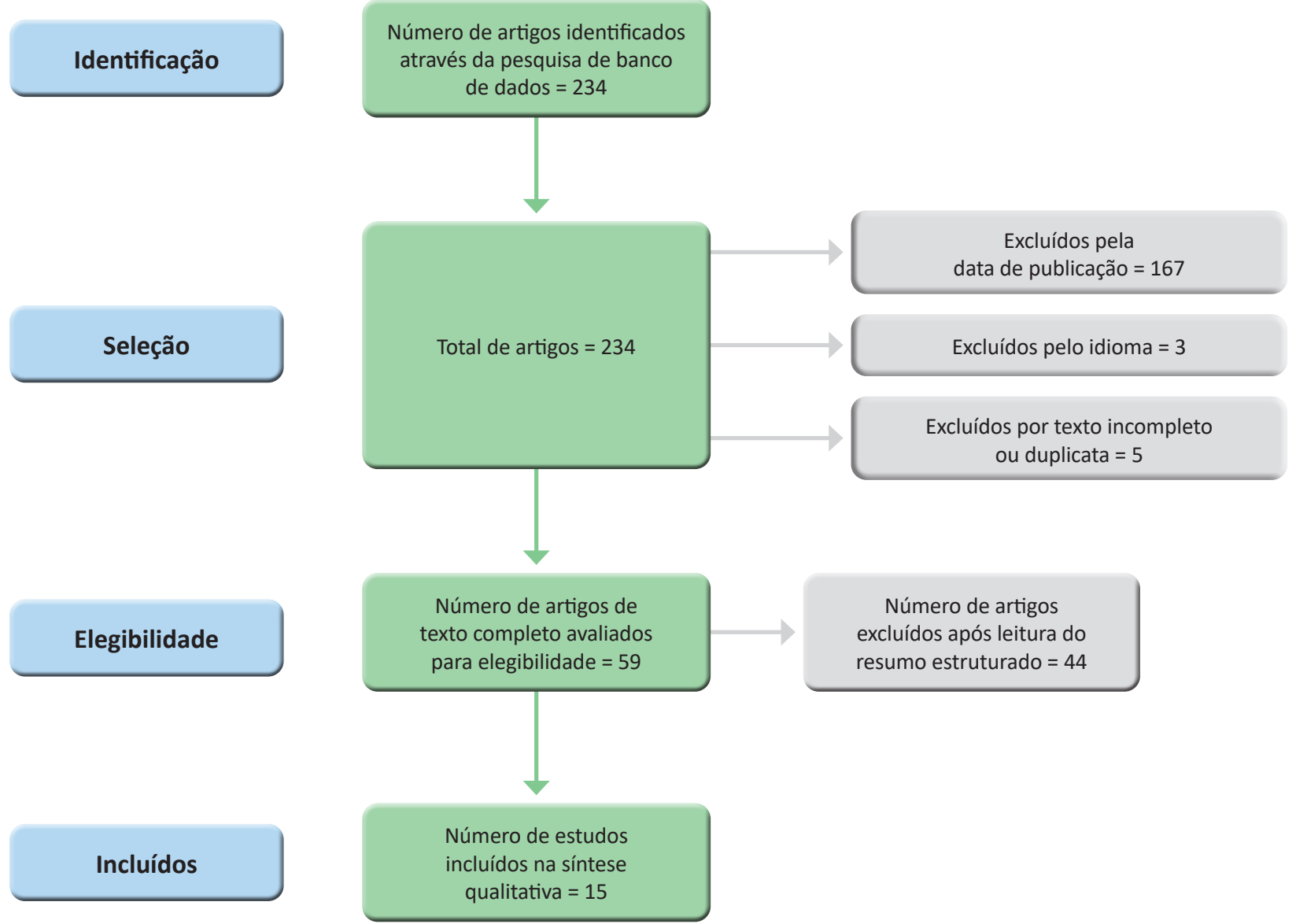


utilização do medicamento; além do mais, estes erros podem estar relacionados, também, com os procedimentos e prática profissional, sendo eles: prescrição, comunicação de pedido, rotulagem, dispensa, distribuição, administração e adesão do paciente; o segundo item, é considerado um fator não evitável e que sempre produz dano ao paciente $(10,11)$.

Diante disso, o farmacêutico tem sido reconhecido como um dos profissionais elementares dentro dos serviços de saúde, principalmente ao serem inseridos no processo de uso dos medicamentos, na equipe de cuidados de saúde. Atuando com essa equipe, o farmacêutico proporciona uma melhor qualidade no atendimento, pode auxiliar na monitorização do processo medicamentoso, de modo a prevenir os erros relacionados a esse processo, assegurando uma farmacoterapia de qualidade ao paciente (12).

Atuação do farmacêutico clínico na identificação de PRM em hospitais. Os PRM podem gerar altos custos no serviço de saúde, assim como podem ocasionar outros transtornos, tais como mudança no resultado terapêutico dos pacientes, problemas psicológicos a estes e aos profissionais envolvidos e podem aumentar os índices de morbimortalidade. Com base nisso, investir em ferramentas e nas competências da profissão farmacêutica é fundamental para prevenir esses problemas, além de favorecerem no processo de tomada de decisão sobre a farmacoterapia envolvida e promover uma cultura de segurança ao paciente (13).

Os resultados do estudo feito por Lima e cols. (2016), que trata sobre a orientação farmacêutica na alta hospitalar, demonstraram que o $\mathrm{FC}$, junto com sua equipe de farmacêuticos residentes, era responsável pelas orientações sobre o tratamento medicamentoso de cada paciente (14). Para a correta orientação, eram avaliados diversos parâmetros como a prescrição, o modo correto de administração e armazenamento dos medicamentos, o horário de tomada do medicamento, a possibilidade de interações medicamentosas ou RAM, além de dialogarem com os pacientes sobre a importância da adesão ao tratamento.

Nesse contexto, a função principal do FC é de proporcionar uma orientação adequada do tra- tamento medicamentoso, devendo repassar tais orientações não apenas de forma verbal, como também, utilizando instrumentos que possam facilitar o entendimento do paciente com relação ao que está sendo orientado, como por exemplo, por meio do uso de símbolos, figuras, cores, dentre outros (10).

O estudo realizado por Lombardi e cols. (2016) mostrou a ocorrência de divergências encontradas na adaptação medicamentosa de pacientes admitidos em unidades de cardiologia, num hospital de grande porte. Foram apontadas 866 divergências, sendo elas por omissão, dose, intervalo e via de administração; destas, 97\% foram resolvidas após a intervenção do FC, prevenindo possíveis consequências clínicas importantes (15).

Com base nesses resultados, os autores destacaram a importância da adaptação medicamentosa correta no momento da admissão de um paciente, pois, essa ação diminui a disparidade que há entre os medicamentos consumidos na residência do mesmo e os prescritos durante a internação. $\mathrm{O}$ farmacêutico, portanto, é um profissional indispensável no que tange a análise da terapia medicamentosa, sendo importante a intervenção do mesmo para propor soluções no caso de ocorrência dessas divergências entre medicamentos (15).

O levantamento realizado por Okumura e cols. (2016), no hospital de Campo Largo, onde o Serviço de Farmácia Clínica (SFC) foi implantado desde 2012, abordou a evolução do SFC no Brasil, dando ênfase às atividades clínicas desenvolvidas pelos farmacêuticos. Esse serviço consistia em: participação em visitas clínicas, elaboração de protocolos institucionais, Monitoração Terapêutica de Fármacos (MTF) antiepiléticos, revisão de cada uma das dosagens dos medicamentos prescritos, as indicações, duração do tratamento, as interações medicamentosas, as contraindicações, entre outros (16).

O resultado do estudo feito pelos autores supracitados, mostrou que a atuação do farmacêutico é imprescindível para reduzir a morbidade relacionada a medicamentos, por meio de suas intervenções, como por exemplo, ajuste das doses, verificação da compatibilidade e estabilidade das preparações prescritas, verificação das concentrações séricas 
dos medicamentos e verificação das alterações de peso dos pacientes que podem influenciar na eficácia do tratamento. Essas ações são consideradas de suma importância na qualidade dos serviços prestados pelos hospitais e consequentemente, pelo SFC (16).

Isso é ratificado pelo objetivo do SFC, que é de padronizar o atendimento à saúde, solucionando os PRM e propondo intervenções farmacêuticas eficazes que vão colaborar com a farmacoterapia e consequentemente, com a saúde do paciente. Além disso, muitos hospitais de grande porte com SFC implementado, visam a detecção de RAM, a prevenção dos PRM evitáveis e minimizar os eventos indesejados relacionados a medicamentos (17)

No estudo realizado por Santos e cols. (2019) foi avaliada a implantação da reconciliação medicamentosa em um hospital, com a utilização de um sistema eletrônico, juntamente com a equipe multiprofissional do referido estabelecimento. Os autores ressaltaram que em um hospital, o trabalho deve ser compartilhado por todos os profissionais envolvidos no cuidado ao paciente; os farmacêuticos, por sua vez, são responsáveis pela conferência dos medicamentos registrados no sistema concomitante com a análise da prescrição realizada pelo médico (18).

De acordo com os dados analisados, o farmacêutico deve verificar se a dose, o intervalo de administração e a via de administração do medicamento estão de acordo com o que foi registrado no histórico do paciente, a fim de identificar possíveis discrepâncias no tratamento dos pacientes. Essas discrepâncias podem ser intencionais ou não: a primeira ocorre quando um medicamento é prescrito de forma diferente daquela que o paciente vinha utilizando, porém o prescritor o faz intencionalmente, por algum motivo específico e de modo deliberado; a segunda ocorre quando a prescrição de um medicamento é alterada no momento da admissão, contudo, o prescritor o faz sem intenção, podendo ser por descuido ou por desconhecimento no ato da admissão (18).

$\mathrm{O}$ acompanhamento diário por parte do $\mathrm{FC}$, assim como a criação de medidas para o cumprimento da reconciliação de medicamentos uti- lizados pelos pacientes, pode contribuir para maior segurança quanto ao uso de medicamentos nos hospitais, minimizando os riscos relacionados aos PRM, melhorando a terapia dos pacientes e seus resultados e, oferecendo qualidade no atendimento (7).

PRM mais comuns em hospitais e as intervenções farmacêuticas realizadas. Os resultados do estudo realizado por Aguiar e cols. (2018) mostraram que os PRM mais identificados foram com relação ao EM, sendo eles: prescrição incompleta, subdosagem, problema farmacocinético requerendo ajuste de dose, sobredose, tempo de infusão incorreto, prescrição duplicada, via de administração inapropriada e regime de dose com frequência menor que o recomendado; tais PRM contribuíram para gerar um custo elevado para o hospital. Todavia, após intervenção farmacêutica, sobretudo a inclusão de informações omissas, alteração de dose e cancelamento da prescrição, e com os ajustes relacionados a esses PRM, foi apontada uma diminuição dos custos para o hospital, resultando em uma aceitação de $98 \%$ dessa intervenção farmacêutica, por parte da equipe médica (3).

A aceitabilidade por parte da equipe médica pôde ser constatada também no estudo realizado por Yiasmam e cols. (2020), onde o percentual de aceitação das IF realizadas foi de $92 \%$ e as intervenções mais efetuadas pelos farmacêuticos foram os ajustes de dosagens, inclusão de terapia adicional e a não-adesão ao tratamento. Esse estudo foi feito entre pacientes com câncer infantil da Etiópia, onde os autores relatam que tratar esse tipo de doença ainda é um dos mais complexos desafios enfrentados pela medicina, pois, está associado a muitos PRM, como por exemplo, os efeitos adversos, EM, interações medicamentosas e não adesão ao tratamento, fatores estes que foram identificados no estudo em questão (19).

Nunes e cols. (2017), dos PRM identificados, $84 \%$ foram relacionados a efetividade do tratamento e $15 \%$ a RAM, sendo que a maior causa desses problemas estava na escolha da dose do medicamento utilizado, a saber, os aminoglicosídeos. A solução dessa questão passou pela necessária monitorização periódica por parte do FC, o qual fez o emprego racional desse fármaco, baseando-se 
na escolha adequada e em esquemas posológicos criteriosos; tal intervenção obteve alta aceitabilidade pelos médicos, com reconhecimento de que o trabalho exercido pelo FC promove benefícios, tais como: redução dos erros de prescrição, redução dos eventos adversos evitáveis e melhor resultado na farmacoterapia empregada (20).

No levantamento realizado por Nascimento e cols. (2020), foram identificados PRM em neonatos, os quais $49 \%$ estavam relacionados a efetividade do tratamento, $46 \%$ a RAM e $1 \%$ foram referentes a custos do tratamento. As intervenções promovidas pelos farmacêuticos, denominadas de "recomendações farmacêuticas", foram direcionadas aos médicos e aos enfermeiros do hospital, obtendo aprovação de 92\%; essas recomendações foram fundamentais para reconhecer que o SFC é uma estratégia necessária para prevenir e reduzir os PRM (21).

Complementando esses estudos, Volpe e cols. (2016) defenderam que a utilização de sistemas eletrônicos de prescrição que podem auxiliar na redução de PRM, nos seguintes aspectos: ilegibilidade, prescrição com nome comercial e presença de informações importantes que favorecem uma prescrição eficaz e segura. Para isso, o farmacêutico precisa dispor de habilidade técnica para utilizar adequadamente esse tipo de sistema informacional (22).

A competência do FC em operar sistemas informatizados de prescrição médica pode estar relacionada com o nível de aceitabilidade dos médicos com relação às intervenções realizadas pelos farmacêuticos. Segundo Souta e cols. (2016), a taxa de aceitação dos médicos em relação às intervenções foi de $47 \%$ apenas, mas, isso se deu pela ausência do FC na revisão das prescrições que são de responsabilidade do mesmo (23).

Araújo e cols. (2017) defenderam o uso do sistema de prescrição eletrônica sob a condição de supervisão do farmacêutico, visto que, em alguns casos, pode ocorrer ausência de informações importantes que vão influenciar tanto no preparo quanto na administração do medicamento. O FC, nesse contexto, por meio da análise dessa prescrição, vai observar tais erros e corrigi-los, de modo a evitar danos à saúde dos pacientes (4). Esses sistemas devem conter dados que possam auxiliar tanto no acompanhamento farmacoterapêutico quanto nos cuidados prestados por outros profissionais, mantendo uma comunicação eficaz relacionada ao tratamento do indivíduo. Ainda, evolução, condutas e condições clínicas de cada paciente devem estar claras nesses sistemas, permitindo a comunicação integral a todos os profissionais envolvidos nesse processo, facilitando a solução dos problemas que surgem (4).

\section{CONCLUSÃO}

A profissão farmacêutica tem passado por várias atualizações, permitindo ao profissional atuar em diversas vertentes. A identificação de PRM é uma tarefa não só do Farmacêutico Clínico (FC), como também de médicos e enfermeiros; porém, em virtude de sua competência e habilidade, é imprescindível a participação do $\mathrm{FC}$ em hospitais, principalmente por ter sensibilidade profissional para captar esses problemas.

Desse modo, o FC atua diretamente em conciliação com a equipe médica verificando o tratamento medicamentoso, avaliando as prescrições e repassando informações relevantes para prevenção de problemas na saúde do paciente. É responsável por orientar e facilitar o entendimento dos pacientes quanto ao tratamento que vão receber, podendo utilizar-se de ferramentas que auxiliem nesse processo. O acompanhamento farmacêutico é de suma importância, pois promove a segurança do paciente e a qualidade do serviço prestado. 
1. Cruz PF, Paiva AC, Pereira Junior MS, Muniz JJ. Problemas relacionados aos medicamentos em pacientes atendidos no pronto socorro de um hospital universitário. Infarma - Cienc. Farm. 2020;32(4):383-390. DOI: 10.14450/2318-9312

2. PCNE. Classification of drug related problems. Pharmaceutical Care Network Europe. 2010 Disponível em: http://www.pcne.org/upload/files/11 PCNE_classification_V6-2.pdf.

3. Aguiar KS, Santos JM, Cambrussi MC, Picolotto S, Carneiro MB. Segurança do paciente e o valor da intervenção farmacêutica em um hospital oncológico. Eisntein. 2018;16(1):1-7.

4. Araújo EO, Viapiana M, Domingues EAM, Oliveira GS, Polisel CG. Intervenções farmacêuticas em uma unidade de terapia intensiva de um hospital universitário. Rev Bras Farm Hosp Serv Saúde. 2017;8(3):25-30. DOI: 10.30968/rbfhss.2017.083.005

5. Ribeiro VF, Sapucaia KCG, Aragão LAO, Bispo ICS, Oliveira VF, Alves BL. Realização de intervenções farmacêuticas por meio de uma experiência em farmácia clínica. Rev Bras Farm Hosp Serv Saúde. 2015; 6(4):18-22.

6. Gomes AD, Galato D, Silva EV. Erros de prescrição de medicamentos potencialmente perigosos em um hospital terciário. Rev Bras Farm Hosp Serv Saúde. 2017;8(3):4247.

7. Souza LB, Souza DM, Souza SM, Silva DR, Aguilar NC. Importância do farmacêutico clinico no uso seguro e racional de medicamentos no âmbito hospitalar. Rev Pens Acad. 2018;16(1):109-124. DOI: 10.21576/ rpa.2018v16i1.360

8. Rodrigues JPV, Pereira LRL. Farmácia clínica em ambiente hospitalar: perspectivas e estratégias para implementação. J.App.Pharm.Sci. 2016;3(S1):7-10.

9. Moher D, Shamseer L, Clarke M, Ghersi D, Liberati A, Petticrew M, Shekelle P, Stewart LA. Preferred Reporting Items for Systematic Review and MetaAnalysis Protocols (PRISMA-P). Syst Rev. 2015;4(1): 1-25. DOI: $10.1136 /$ bmj.g7647

10. . Ferreira Neto CJB, Plodek CK, Soares FK, Andrade RA, Teleginski F, Rocha MD. Intervenções farmacêuticas em medicamentos prescritos para administração via sondas enterais em hospital universitário. Rev. LatinoAm. Enferm. 2016;24:1-9. DOI: 10.1590/1518-8345. 0619.2696

11. Souza AFR, Queiroz JC, Vieira AA, Solon LGS, Bezerra ELSF. Os erros de medicação e os fatores de risco associados a sua prescrição. Enferm. Foco. 2019;10(4):12-16.

12. Cruz HL, Mota FKC, Araujo LU, Bodevan EC, Seixas SRS, Santos DF. A utilidade do registro médico: fatores associados aos erros de medicamentos em pacientes com doenças crônicas. Rev. Latino-Am. Enferm. 2017;25: 1-10. DOI: 10.1590/1518-8345.2406.2967
13. Vilela RPB, Jericó MC. Implantação de tecnologias para prevenção de erros de medicação em hospital de alta complexidade: análise de custos e resultados. Einstein. 2019;17(4):1-7.DOI: 10.31744/einstein_journal/2019GS 4621

14. Lima LF, Martins BCC, Oliveira FRP, Cavalcante RMA, Magalhães VP, Firmino PYM, Adriano LS, Silva AM, Flor MJN, Néri EDR. Orientação farmacêutica na alta hospitalar de pacientes transplantados: estratégia para a segurança do paciente. Einstein. 2016;14(3):359-365. DOI: $10.1590 / \mathrm{S} 1679-45082016 \mathrm{AO} 3481$

15. Lombardi NF, Mendes AEM, Lucchetta RC, Reis WCT, Favero MLD, Correr CJ. Análise das discrepâncias em unidades de cardiologia: um estudo descritivo. Rev. Latino-Am. Enferm. 2016;24(1):1-7. DOI: 10.1590/ $1518-8345.0820 .2760$

16. Okumura LM, Silva DM, Comarella, L. Relação entre o uso seguro de medicamentos e serviços de farmácia clínica em unidades de cuidados intensivos pediátricos. Rev Paul Pediatr. 2016;34(4):397-402.

17. Bhatt-Mehta V, Buck ML, Chung AM, Farrington EA, Hagemann TM, Hoff DS, LaRochelle JM, Pettit RS, Phan H, Potts A, Smith KP, Parrish Second HR. Recommendations for meeting the pediatric patient's need for a clinical pharmacist: a joint opinion of the pediatrics practice and research network of th eamerican college of clinical pharmacy and the pediatric pharmacy advocacy group. J Pediatr Pharmacol Ther. 2012;17(3):281-291. DOI: $10.1002 /$ phar. 1246

18. Santos CO, Lazaretto FZ, Lima LH, Azambuja MS, Millão LF. Reconciliação de medicamentos: processo de implantação em um complexo hospitalar com a utilização de sistema eletrônico. Saúde Debate. 2019;43(121): 368-377. DOI: 10.1590/0103-1104201912106

19. Yismaw MB, Adam H, Engidawork E. Identification and resolution of drug-related problem among childhood cancer patients in Ethiopia. J Clin Oncol. 2020;(2):1-9. DOI: $10.1155 / 2020 / 6785835$

20. Nunes BM, Xavier TC, Martins RR. Problemas relacionados a medicamentos antimicrobianos em unidade de terapia intensiva neonatal. Rev Bras Ter Intensiva. 2017;29(3):331-336. DOI: 10.5935/0103507x.20170040.

21. 21. Nascimento ARF, Leopoldino RWD, Santos MET, Costa TX, Martins RR. Problemas relacionados a medicamentos em neonates cardiopatas sob terapia intensiva. Rev Paul Pediatr. 2020;38(1):1-6. DOI: 10.1590/1984-0462/2020/38/2018134

22. Volpe CRG, Melo EMM, Aguiar LB, Pinho DLM, Stival MM. Fatores de risco para erros de medicação na prescrição eletrônica e manual. Rev. Latino-Am. Enferm. 2016;24:1-9. DOI: 10.1590/1518-8345.0642.2742

23. Souta MM, Telles Filho PCP, Vedana KGG, Pedrão LJ, Miasso AI. Sistema de medicação: análise das ações dos profissionais em unidades de internação psiquiátrica. Texto contexto - Enferm. 2016;25(4):1-9. DOI: 10.1590/ 0104-07072016000170015 